\title{
Mixed messages: modulation of inflammation and immune responses by prostaglandins and thromboxanes
}

\author{
Stephen L. Tilley, ${ }^{1}$ Thomas M. Coffman, ${ }^{2}$ and Beverly H. Koller ${ }^{1}$ \\ ${ }^{1}$ Division of Pulmonary and Critical Care Medicine, Department of Medicine, University of North Carolina-Chapel Hill, \\ Chapel Hill, North Carolina, USA \\ ${ }^{2}$ Division of Nephrology, Department of Medicine, Duke University and Durham Veterans Affairs Medical Centers, \\ Durham, North Carolina, USA \\ Address correspondence to: Beverly H. Koller, 7027 Thurston-Bowles Building, University of North Carolina-Chapel Hill, Chapel Hill, \\ North Carolina 27599-7248, USA. Phone: (919) 962-2153; Fax: (919) 966-7524; E-mail: Treawouns@aol.com. \\ J. Clin. Invest. 108:15-23 (2001). DOI:10.1172/JCI200113416.
}

Virtually every organism has evolved mechanisms by which, upon stimulation, lipids are released from plasma membranes and metabolized into mediators capable of changing cellular physiology. As these lipids are present at the first site exposed to external challenge, they provide an ideal substrate for the synthesis of defensive mediators and homeostatic regulators. One such group of lipid mediators is the prostanoids, including the prostaglandins (PGs) and thromboxanes (TXs).

Soon after their initial isolation and characterization, the ability of prostanoids to influence inflammation and immune responses was recognized. For example, administration of prostanoids, either alone or in combination, could reproduce the cardinal signs of inflammation. Because they could induce inflammatory changes when injected into tissue and were present at high levels in inflamed lesions, prostanoids were initially categorized as proinflammatory mediators. As our understanding of prostanoid physiology has evolved, it has become clear that these mediators can act to both promote and inhibit inflammation. Thus, it is more accurate to envision these molecules as part of a complex regulatory network that modulates the actions of immune cells and the surrounding microenvironment. Their overall impact in an individual inflammatory response will depend on several factors, including the level of immune cell activation, the presence of other mediators, and the physiological state of the organism.

Our ability to dissect the role of prostanoids in complex inflammatory responses has been substantially advanced by the recent development of mouse lines with targeted mutations of genes encoding enzymes and receptors in the prostanoid pathway. In this review we will develop the concept that prostanoids are both effectors and regulators of inflammation, emphasizing new information provided by these mouse models.

Production of prostanoids during inflammation Prostanoids are produced when arachidonic acid (AA) is released from the plasma membrane by phospholipases and metabolized by cyclooxygenases (COXs) and specific isomerases. During an inflammatory response, both the level and the profile of prostanoid production can change dramatically. While prostanoid levels are generally very low in uninflamed tissues, they increase
Table 1

Inflammatory responses in mice deficient in prostanoid synthetic enzymes and receptors

\begin{tabular}{lccc}
\hline Model & Disrupted gene & Inflammatory response & Reference \\
AA-induced ear edema & COX-1 & $\downarrow$ & 46 \\
& COX-2 & $\leftrightarrow$ & 47,48 \\
& EP3 & $\downarrow$ & J.L. Goulet et al., manuscrip \\
& & & submitted for publication \\
TPA-induced ear edema & COX-1 & $\leftrightarrow$ & 46 \\
& COX-2 & $\leftrightarrow$ & 47,48 \\
Carrageenan air pouch & COX-1 & $\downarrow$ at $3 \mathrm{~d}$ & 30 \\
& COX-2 & $\downarrow \downarrow$ at $3 \mathrm{~d}$ & 49 \\
& COX-1 & $\leftrightarrow$ at $7 \mathrm{~d}$ & 30 \\
Carrageenan paw edema & COX-2 & $\uparrow$ at $7 \mathrm{~d}$ & 30 \\
& COX-2 & $\leftrightarrow$ & 49 \\
Carrageenan pleurisy & IP & $\downarrow$ & 32 \\
TNF/D-galactosamine & IP & $\downarrow$ & 32 \\
hepatocellular toxicity & COX-2 & $\downarrow$ & 48 \\
Allergic airway disease & COX-1 & $\uparrow \uparrow$ & 43 \\
& COX-2 & $\uparrow$ & 43 \\
& DP & $\downarrow$ & 45 \\
Dextran sulfate colitis & COX-1 & $\uparrow$ & 42 \\
Collagen-induced arthritis & COX-2 & $\uparrow \uparrow$ & 42 \\
& COX-1 & $\leftrightarrow$ & 41 \\
& COX-2 & $\downarrow$ & 41
\end{tabular}

TPA, tetradecanoyl phorbol acetate. 
Table 2

Expression of prostanoid synthases and receptors on immune cells

\begin{tabular}{|c|c|c|c|c|c|c|c|c|c|}
\hline Synthase & Thymus & Spleen & T lymphocyte & B lymphocyte & $\begin{array}{l}\text { Dendritic } \\
\text { cell }\end{array}$ & $\begin{array}{l}\text { Macrophage/ } \\
\text { Monocyte }\end{array}$ & Eosinophil & Neutrophil & $\begin{array}{c}\text { Mast cell/ } \\
\text { Basophil }\end{array}$ \\
\hline PGD & $\mathrm{P}(\mathrm{m}, \mathrm{r})$ & $P(r)$ & $M(h)$ & & $A(r)$ & $A, P(h, r)$ & $\mathrm{P}(\mathrm{h})$ & & $\mathrm{P}, \mathrm{M}(\mathrm{h}, \mathrm{r}, \mathrm{m})$ \\
\hline PGE & $\mathrm{P}(\mathrm{m})$ & $M(r)$ & & & $P(h)$ & $P(h, m)$ & $P(h, g p)$ & $P(h)$ & $A(r)$ \\
\hline PGF & $\mathrm{P}(\mathrm{m})$ & & & & & & $\mathrm{P}(\mathrm{h})$ & & \\
\hline PGI & & & & & & $\mathrm{M}(\mathrm{m})$ & & & \\
\hline TXA & $\mathrm{P}, \mathrm{A}(\mathrm{m}, \mathrm{h})$ & & & & $A(h)$ & $\mathrm{P}, \mathrm{M}, \dot{\mathrm{A}}(\mathrm{h}, \mathrm{m})$ & $P(h, g p)$ & $P(h)$ & $M, P(h)$ \\
\hline \multicolumn{10}{|l|}{ Receptor } \\
\hline CRTH2 & & & $M(h)$ & & & $M(h)$ & $M(h)$ & & $M(h)$ \\
\hline DP & & & & & & & & $\mathrm{P}(\mathrm{h})$ & $P(r)$ \\
\hline EP1 & & $M(r)$ & $\mathrm{M}(\mathrm{m})$ & $\mathrm{M}(\mathrm{h}, \mathrm{m})$ & & $\mathrm{M}(\mathrm{m})$ & & & $\mathrm{M}(\mathrm{m})$ \\
\hline EP2 & $\mathrm{M}(\mathrm{m})$ & $\mathrm{M}(\mathrm{r}, \mathrm{m})$ & $M(m)$ & $M(h, m)$ & & $\mathrm{M}(\mathrm{m})$ & $\mathrm{M}, \mathrm{P}(\mathrm{h}, \mathrm{gP})$ & $P(h, r)$ & $\mathrm{P}, \mathrm{M}(\mathrm{r}, \mathrm{m})$ \\
\hline EP3 & $M(m)$ & $M(r, m)$ & $\mathrm{M}(\mathrm{m})$ & $\mathrm{M}(\mathrm{h}, \mathrm{m})$ & & $M(m)$ & & & $M(m)$ \\
\hline EP4 & $M(m)$ & $M(m)$ & $\mathrm{A}, \mathrm{M}(\mathrm{h}, \mathrm{m})$ & $M(h, m)$ & & $\mathrm{A}, \mathrm{M}(\mathrm{h}, \mathrm{m})$ & & & $M(m)$ \\
\hline \multicolumn{10}{|c|}{ (1) } \\
\hline IP & $\mathrm{M}(\mathrm{m})$ & $\mathrm{M}(\mathrm{m})$ & $\mathrm{P}(\mathrm{h})$ & & & & & & $P(r)$ \\
\hline TP & $M(m)$ & $M(m)$ & $\mathrm{M}(\mathrm{m})$ & & & $\mathrm{P}(\mathrm{h})$ & $\mathrm{P}(\mathrm{h})$ & & \\
\hline
\end{tabular}

A, antibody; M, molecular; P, pharmacology; h, human; m, mouse; r, rat; gp, guinea pig.

immediately in acute inflammation prior to the recruitment of leukocytes. As immune cells infiltrate the tissues, further increases in prostanoid levels are observed. The profile of prostanoids that are produced can also vary dramatically during the course of the response. For example, in the carrageenan-induced pleurisy model, elevated $\mathrm{PGE}_{2}$ levels are observed only during the early stages of inflammation, whereas $\mathrm{PGD}_{2}$ becomes pronounced during the final stages of the response (1).

Prostanoid production depends on the activity of the two COX isoenzymes within cells. COX-1 is present in most cells and its expression is generally constitutive. In contrast, COX-2 expression is low or undetectable in most cells but its expression increases dramatically upon stimulation, particularly in cells of the immune system (2). The recruitment of leukocytes and the induction of COX-2 expression by inflammatory stimuli likely account for the high levels of prostanoids found in chronic inflammatory lesions and provided a rational basis for the development of COX-2-specific inhibitors for treating arthritis and other chronic inflammatory diseases. However, the capacity for COX-1 to modulate inflammatory responses should not be overlooked.

Studies of circulating monocytes after exposure to LPS suggest that some increases in COX-1 expression can occur upon activation (3). In addition, studies using mice deficient in the expression of COX-1 or COX-2 have identified unique roles for COX-1 in the initiation of certain inflammatory responses (Table 1). In mast cells, Reddy and associates found that the production of $\mathrm{PGD}_{2}$ within the first 30 minutes of stimulation depends almost entirely on COX-1. While COX1 also contributes to the production of $\mathrm{PGD}_{2}$ by stimulated mast cells over the next 2-4 hours, maximal production at these later time points is due to upregulation of COX-2 expression (4). Thus, the earliest prostanoid response to deleterious environmental stimuli is dependent on COX-1, and as the inflammatory process progresses, COX-2 becomes a major source of prostanoids.

$\mathrm{PGH}_{2}$ is produced by both COX isoforms and is the common substrate for a series of specific synthase enzymes that produce $\mathrm{PGD}_{2}, \mathrm{PGE}_{2}, \mathrm{PGF}_{2 \alpha}, \mathrm{PGI}_{2}$, and $\mathrm{TXA}_{2}$. It is the differential expression of these enzymes within cells present at sites of inflammation that will determine the profile of prostanoid production (Table 2). For example, mast cells predominantly generate $\mathrm{PGD}_{2}$ while macrophages produce $\mathrm{PGE}_{2}$ and $\mathrm{TXA}_{2}$. In addition, alterations in the profile of prostanoid synthesis can occur upon cellular activation. While resting macrophages produce $\mathrm{TXA}_{2}$ in excess of $\mathrm{PGE}_{2}$, this ratio changes to favor $\mathrm{PGE}_{2}$ production after LPS activation. Several biochemical mechanisms have been proposed to explain this altered synthetic profile. First, it has been suggested that physical compartmentalization of COX-1 or COX-2 with specific terminal synthases could link the activity of these enzymes with the synthesis of specific prostanoid end products (5). Second, some of the synthases are inducible, and their expression may be regulated by environmental signals. For example, expression of the glutathione-dependent isoform of PGE-synthase is enhanced by IL-1 $\beta$ (6). Finally, it has been suggested that differences in substrate affinity and kinetics of PGE-synthase and TXA-synthase account for different production profiles of resting and activated monocytes (7). Thus, the quantity and variety of prostanoids that are produced during inflammation are deter- 
mined by the nature and the activation state of the cells present in the inflammatory lesion.

\section{Receptors for prostanoids}

The ability of a given prostanoid to affect immune cell function depends on its binding to $G$ protein-coupled cell surface receptors. The actions of $\mathrm{PGF}_{2 \alpha}, \mathrm{PGI}_{2}$, and $\mathrm{TXA}_{2}$ are mediated by individual receptors, the FP, IP, and TP receptors, respectively. In contrast, $\mathrm{PGD}_{2}$ and $\mathrm{PGE}_{2}$ activate multiple receptors. $\mathrm{PGD}_{2}$ acts through two receptors, the DP receptor and the recently identified CRTH2 receptor (chemoattractant receptorhomologous molecule expressed on Th2) (8). There are four subtypes of receptors for $\mathrm{PGE}_{2}$, termed EP1-EP4, each encoded by a distinct gene. Because the repertoire of receptors expressed by various immune cell populations differs, the function of these cells is modified differently by the prostanoids present in the local environment during an immune response. Table 2 summarizes the expression of prostanoid receptors on immune cells and is based on both pharmacological studies and examination of mRNA.

Prostanoid receptors couple to a range of intracellular signaling pathways that mediate the effects of receptor activation on leukocyte function. The DP, EP2, EP4, IP, and one isoform of the EP3 receptor can couple to $\mathrm{G}_{\mathrm{s}}$ and thus increase intracellular cAMP concentration. In $\mathrm{T}$ cells and other inflammatory cells, cAMP accumulation is generally associated with inhibition of effector cell functions. EP1, FP, IP, and TP receptors, as well as other EP3 isoforms, couple to $\mathrm{G}_{\mathrm{q}}$, and activation of these receptors leads to increased intracellular calcium levels and thus to immune cell activation. Finally, TP, CRTH2, and yet another EP3 receptor isoform can each couple to $G_{i}$, causing cAMP levels to decline while also mobilizing intracellular calcium. As shown in Table 2, many cells of the immune system express multiple receptors that couple to these apparently opposing pathways. As one example, immature bone marrow-derived mast cells express EP2, EP3, and EP4 receptors. Stimulation of EP2 and EP4 would be expected to inhibit mast cell function while stimulation of the EP3 receptor would increase intracellular calcium and/or inhibit cAMP and thereby promote mast cell degranulation. We still have limited understanding of how these potentially conflicting signals, triggered by the same ligand in the same cell, are integrated and coordinated.

The regulation of the EP2 and EP4 receptors provides additional examples of mechanisms by which cells can modify their responses to prostanoids. Although both receptors bind $\mathrm{PGE}_{2}$ with equal affinity and both are coupled to $G_{s}$, a number of observations indicate that they do not mediate similar cellular functions. Rather, subtle differences between these receptors may provide a means of fine-tuning the organism's response to extracellular $\mathrm{PGE}_{2}$. First, while both the EP2 and the EP4 receptors are expressed in most tissues, the level of expression of the EP4 receptor in most cells is higher (9). However, large increases in EP2 receptor expression are observed under specific physiological conditions. For example, EP2 receptor expression is induced in the uterus just after embryo implantation, and up to fivefold increases in the expression of EP2 have been observed in LPS-stimulated macrophages (9). In contrast, these macrophages show only a slight increase in EP4 levels, indicating that EP2 and EP4 are also under independent regulation. Moreover, these receptors differ in their rate of desensitization and their ability to bind 15-keto-PGE 2 , the primary metabolite of $\mathrm{PGE}_{2}$ (ref. 10; and D. Slipetz, personal communication). The unique functions of these $\mathrm{G}_{\mathrm{s}}$-coupled receptors are highlighted by the lack of overlap in the phenotypes of EP2- and EP4-deficient mice (11-13). Examination of the phenotype of mice deficient in both receptors should determine whether cooperation between these receptors occurs in some physiological settings.

In summary, the impact of prostanoids present during an inflammatory response on both immune effector cells and surrounding stromal cells is determined by the array of receptors the cells express and the intracellular pathways to which they are coupled. Activation of these receptors, even when coupled to similar pathways, might evoke different responses because of different levels of expression (both constitutive and induced), different patterns of desensitization, and differential affinity to metabolites of the primary ligand. Thus, determining the role of prostanoids in a given inflammatory response requires not only knowledge of the lipid mediators present in the lesion, but also the receptor profile on immune cells and the biochemical signaling of these receptors under specific ligand concentrations. Figure 1, which indicates potential proand anti-inflammatory roles of $\mathrm{PGE}_{2}$ and $\mathrm{TXA}_{2}$ in asthma, highlights these complexities.

\section{Lymphocyte-prostanoid interactions}

The T lymphocyte is responsible for the initiation and coordination of immune responses and thus plays a pivotal role in triggering antigen-specific inflammation. Interactions between prostanoids and $\mathrm{T}$ cells have been long recognized and the effects of prostanoids on a range of $T$ cell functions have been described. One action of prostanoids on $\mathrm{T}$ cell function may be the modulation of $\mathrm{T}$ cell development and maturation in the thymus. Both COX isoforms, as well as a number of prostanoid receptors, are expressed in thymus, and accumulating evidence suggests that these components of the prostanoid pathway regulate thymic functions.

TP receptors are expressed at high levels in the thymus, most prominently in immature thymocyte populations, and stimulation of TP receptors on these cells promotes 
a

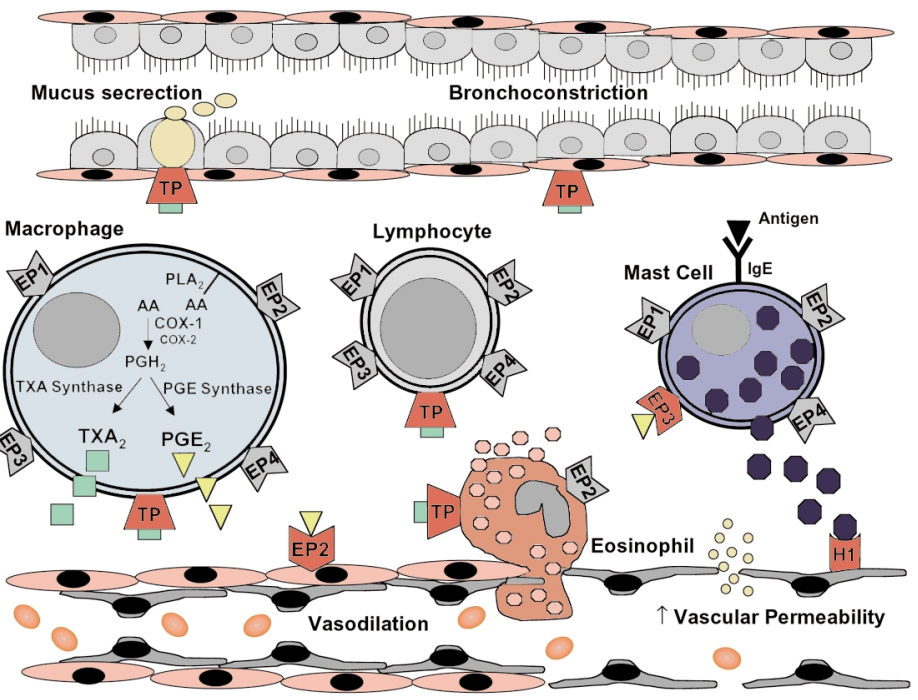

b

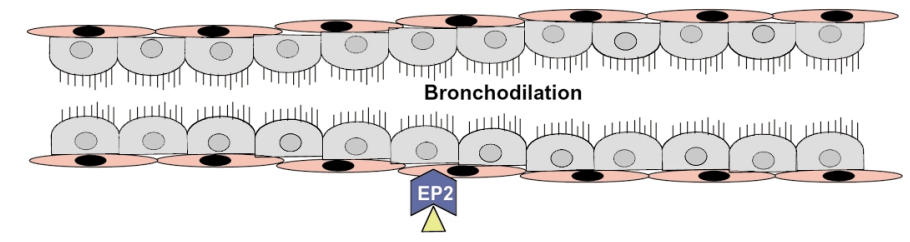

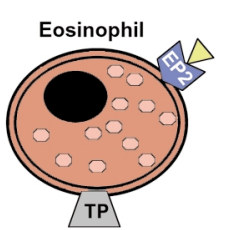

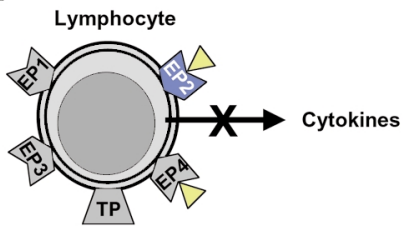

Figure 1

Potential pro- and anti-inflammatory actions of prostanoids in asthma. (a) Proinflammatory actions of $\mathrm{TXA}_{2}$ and $\mathrm{PGE}_{2}$. Solid red receptors indicate pathways that enhance airway inflammation. $\mathrm{TXA}_{2}$ activates leukocytes and increases airway obstruction by inducing airway smooth muscle contraction and goblet cell mucus secretion. $\mathrm{PGE}_{2}$ promotes vasodilation by activating CAMP-coupled EP2 receptors on vascular smooth muscle and increases vascular permeability indirectly by enhancing the release of histamine and other mediators from tissue leukocytes such as mast cells. Receptors for which there is insufficient information to define a proinflammatory role are depicted in gray. (b) Anti-inflammatory actions of $\mathrm{PGE}_{2}$. Solid blue receptors indicate pathways that reduce airway inflammation. As inflammation progresses, $\mathrm{PGE}_{2}$ synthesis by macrophages is enhanced due to increased expression of COX-2 and PGE-synthase. $\mathrm{PGE}_{2}$ inhibits leukocyte activation and promotes bronchodilation through activation of $\mathrm{G}_{\mathrm{s}}$-coupled EP2 and EP4 receptors. Increased expression of EP2 receptors is likely to further enhance these anti-inflammatory actions of $\mathrm{PGE}_{2}$. Receptors for which there is insufficient information to define an anti-inflammatory role are depicted in gray. $\mathrm{H} 1$, histamine receptor; $\mathrm{TP}, \mathrm{TXA}_{2}$ receptor; $\mathrm{EP}, \mathrm{PGE}_{2}$ receptor.

apoptosis (14). These data suggest that TP receptors on thymocytes might play a role in negative selection of maturing $\mathrm{T}$ lymphocytes. These actions may be relevant to in vivo immune responses, since Remuzzi and associates have found that the actions of TP receptors in the thymus may be critical for the development of tolerance to renal allografts (15).

Early studies suggested that $\mathrm{PGE}_{2}$ protects immature $\mathrm{CD}^{+} \mathrm{CD}^{+}$(double positive) thymocytes from apoptosis (16). This action appears to be mediated by cAMP, suggesting involvement of EP2 or EP4 receptors. Recently, Rocca and colleagues have addressed the role of prostanoids in lymphocyte development by using fetal organ cultures (17). Their findings suggest that COX isoenzymes play functionally distinct roles in regulating lymphocyte development in the thymus. COX-2 is expressed in a small subset of stromal cells while low levels of COX-1 can be detected in developing thymocytes. Exposure of the fetal thymus to drugs that primarily inhibit COX-1 results in a small decrease in the number of CD4-CD8 ${ }^{-}$thymocytes that differentiate into double positive cells. A similar decrease in the number of double positive cells has been observed in COX-1-deficient mice. This defect can be rescued by addition of $\mathrm{PGE}_{2}$, or $\mathrm{EP} 2$ agonists to the organ cultures. Similar studies of thymocyte development in mice treated with COX2 -specific inhibitors or in COX-2-deficient animals suggest a role for COX-2 in the very early stages of thymocyte maturation in the differentiation of double positive cells into mature $\mathrm{CD} 4^{+}$thymocytes. While these studies continue to support a role for prostanoids in $\mathrm{T}$ cell development, they do not provide evidence to support a mechanism whereby $\mathrm{PGE}_{2}$ and/or $\mathrm{TXA}_{2}$ regulate apoptosis of immature thymocytes. Additional studies are required to determine whether these subtle differences in $\mathrm{T}$ cell maturation will alter $\mathrm{T}$ cell repertoires in COX-deficient animals.

Alterations in thymocyte differentiation or in the development of other immune cell populations have not yet been reported in the primary characterization of the prostanoid receptor-deficient mice. In these studies, however, lymphocyte development and maturation have largely been evaluated qualitatively by light microscopy, 
immunofluorescence, and cytofluorometry. Thus, while no profound effect on development of lymphoid populations has been observed in healthy unchallenged animals, it remains to be determined whether more substantive alterations may become apparent with experimental stress or on genetic backgrounds that promote autoimmunity.

Prostanoids may also influence the function of mature lymphocytes. For example, $\mathrm{PGE}_{2}$ inhibits a wide range of $\mathrm{T}$ and $\mathrm{B}$ cell functions. These include inhibition of $\mathrm{T}$ lymphocyte activation and proliferation and of $\mathrm{B}$ lymphocyte functions, including Ig production (18-20). Conversely, $\mathrm{TXA}_{2}$ may promote T cell activation and proliferation and facilitate the development of effector cytolytic T cells (21). These findings from pharmacological experiments have generally been confirmed in preliminary studies with EP- and TP-deficient mouse lines using assays of cellular immunity such as the mixed lymphocyte response. Using this experimental approach, the EP2 receptor has been identified as the receptor that mediates the actions of $\mathrm{PGE}_{2}$ to inhibit $\mathrm{T}$ cell proliferation (T.M. Coffman, unpublished results). This finding is also consistent with previous studies that had suggested that the inhibitory effects of $\mathrm{PGE}_{2}$ were related to accumulation of intracellular cAMP, implicating a $\mathrm{G}_{\mathrm{s}}{ }^{-}$ coupled receptor such as EP2 or EP4 (19).

Along with its ability to inhibit $\mathrm{T}$ cell activation and proliferation, $\mathrm{PGE}_{2}$ may also play a role in shaping the immune response. In vitro studies suggest that $\mathrm{PGE}_{2}$ promotes the development of a Th2 response. Interestingly, $\mathrm{PGE}_{2}$ can inhibit the production of the Th1 cytokines IL- 2 and IFN- $\gamma$, shifting the balance in favor of a Th2 response that enhances IL- 4 and IL-5 production and facilitates Ig class switching to $\operatorname{IgE}$ (22). However, other studies have suggested that $\mathrm{PGE}_{2}$ inhibits Th2 cytokine secretion and $\operatorname{IgE}$ production by $B$ cells $(20,23)$. Future studies using mice deficient in EP receptors should clarify the role of $\mathrm{PGE}_{2}$ in T cell-dependent responses in vivo.

\section{G protein-independent actions}

of prostanoids in inflammation

Recent studies suggest two additional pathways through which prostanoids can modify the function of immune cells: by direct activation of nuclear receptors and by inhibitory interactions with intracellular proteins. The suggestion that prostanoids might be ligands for nuclear receptors was inspired by the demonstration that the $\mathrm{PGD}_{2}$ metabolite 15 -deoxy- $\Delta^{12,14}-\mathrm{PGJ}_{2}$ is a potent agonist of the nuclear receptor PPAR $\gamma$ (ref. 23; see also Narumiya and FitzGerald, this Perspective series, ref. 25). This nuclear receptor, which acts as a ligand-dependent transcriptional regulator, was originally identified because of its role in adipocyte differentiation, glucose homeostasis, and control of lipid uptake.
Recent studies have shown that PPAR $\gamma$ is expressed at high levels in activated macrophages. Treatment of freshly prepared peripheral blood monocytes with PPAR $\gamma$ ligands, including 15 -deoxy- $\Delta^{12,14}$ PGJ $_{2}$, decreases cytokine release by activated monocytes (26). Similarly, studies using peritoneal macrophages also showed that 15 -deoxy- $\Delta^{12,14}-\mathrm{PGJ}_{2}$ can modify gene expression. Based on these findings, Ricote and colleagues suggest that activation of PPAR $\gamma$ inhibits the transcription factors AP-1, STAT, and NF-KB (27).

On the other hand, recent studies have also suggested that cyclopentenone PGs such as 15 -deoxy- $\Delta^{12,14}$. $\mathrm{PGJ}_{2}$ directly inhibit the actions of IKB kinase (IKK) (28, 29). This inhibition is mediated by a molecular interaction in which electrophilic carbons from the prostanoid covalently modify the structure of IKK. IKK is responsible for the activation of NF- $\mathrm{KB}$ by proinflammatory stimuli, so its inhibition prevents translocation of NF- $\mathrm{KB}$ to the nucleus and transcription of $\mathrm{NF}-\kappa \mathrm{B}$ target genes. Although the finding that prostanoids can modulate the immune response through mechanisms that are independent of their conventional receptors is exciting and novel, further studies are necessary to establish the relevance and contribution of these pathways to the regulation of inflammatory responses in the intact organism.

\section{Identification of prostanoid pathways modulating} specific immune responses

Pharmacological agents that inhibit COX activity have been used for over 20 years to identify the role of prostanoids in immune responses. More recently, this approach has been complemented by the development of pharmacological inhibitors with specificity for the individual COX isoenzymes and the generation of mice with targeted disruptions of the genes encoding COX1 or COX-2. Since these two enzymes act at the first step in the production of all prostanoids, these studies do not identify the specific prostanoid or the receptor pathway involved. While agonists and antagonists for some of the prostanoid receptors and synthase enzymes have been developed, many of these do not have the requisite affinity or specificities necessary for physiological experiments. The development of mice deficient in each of the prostanoid receptors has therefore provided an important approach for pinpointing the contributions of individual prostanoids and their receptors in inflammation and immune responses.

Acute inflammation. Acute inflammation is the earliest response to tissue injury, infection, or immunological challenge. This physiologic process involves a coordinated response between the immune system and the tissue in which injury has occurred. Prostanoids were implicated in these processes in the 1970s when it was demonstrated that the pharmacologic inhibition of COX by aspirin and other nonsteroidal anti-inflamma- 
tory drugs (NSAIDs) attenuates acute inflammation and that injection of prostanoids into an organism can potentiate many of the signs of inflammation induced by bradykinin and histamine. However, as conventional NSAIDs inhibit both COX isoforms, it was not possible to identify the relative contribution of each COX isoform in these responses. In this regard, mice deficient in COX-1 and COX-2 have been useful for defining the role of COX isoforms in various inflammatory models (Table 1; reviewed in ref. 30). Both COX-1 and COX-2 have been implicated in models of acute inflammation, and it appears that the degree to which each COX isoform contributes depends upon the inflammatory stimulus, the time point examined, and the tissue or organ in which the insult occurs, among other factors.

Application of AA to the surface of the skin elicits an inflammatory response that is entirely dependent on the production of leukotrienes and prostanoids, mimicking the early events in acute inflammation. Because of the substantial contribution of leukotrienes in the AA model, the use of mice deficient in 5-lipoxygenase (5-LO) that are unable to produce leukotrienes provides a means to examine the contribution of prostanoids in isolation. We have found that edema is reduced substantially when 5 -LO-deficient mice are pretreated with the nonselective COX inhibitor indomethacin. Studies in mice with combined 5LO/COX-1 or 5-LO/COX-2 deficiencies show that COX-1 is responsible for the prostanoid component of edema formation in this model (B.H. Koller, unpublished results). These data suggest that the initial inflammatory response to an insult that causes rapid release of AA is mediated by COX- 1 . The findings in this functional assay are consistent with the constitutive expression of high levels of COX-1 in the skin and the observation that early production of prostanoids by activated mast cells depends primarily on COX-1 (31). However, the demonstration of reduced inflammation in other models, using both COX-2-deficient mice and COX-2 inhibitors, suggests that the prostanoids produced after the rapid induction of COX-2 also contribute to acute inflammatory responses (Table 1).

The availability of prostanoid receptor-deficient mice has facilitated the dissection of prostanoid-dependent pathways leading to the various components of the acute inflammatory response. Vasodilation and increased permeability of postcapillary venules, early events in the inflammatory response, reflect the significant effects of PGs on vascular tone at sites of inflammation. Both $\mathrm{PGE}_{2}$ and $\mathrm{PGI}_{2}$ are potent vasodilators in animals and humans and are produced in sufficient quantities at inflammatory sites to account for the characteristic erythema (rubor) of acute inflammation. Both $\mathrm{PGE}_{2}$ and the IP receptor agonist cicaprost produce systemic hypotension when infused intravenous- ly into wild-type mice. In mice deficient in the IP receptor, blood pressure does not change during cicaprost infusion, confirming the role of the IP receptor activation in vasodilation and providing evidence that a single IP receptor is responsible for the action of $\mathrm{PGI}_{2}$ on vascular smooth muscle (32). Because of the existence of multiple EP receptors with different coupling mechanisms, identification of the pathways through which $\mathrm{PGE}_{2}$ mediates changes in blood flow has been more difficult. Initial studies using EP-deficient mice suggested a major role for the EP2 receptor, at least in the systemic circulation (33). However, these studies identified additional complexity in these vascular responses since the relative contribution of individual EP receptors differed substantially between males and females. The specific EP receptors that mediate $\mathrm{PGE}_{2}-$ induced vasodilation in the microcirculation and at sites of inflammation remain undefined.

Along with prostanoids, several other mediators, including histamine, bradykinin, and leukotrienes, influence vascular permeability in models of acute inflammation. In fact, when administered alone, prostanoids produce only small changes in vascular permeability. However, $\mathrm{PGE}_{2}$ and $\mathrm{PGI}_{2}$ can substantially potentiate the effects of bradykinin and histamine on edema formation. In one model of acute inflammation, Murata and colleagues showed that the prostanoid contribution to carrageenan-induced paw edema was entirely due to $\mathrm{PGI}_{2}$ acting through the IP receptor (32). In contrast, studies with all four EP-deficient mouse lines in the AA model have suggested that these actions are mediated by $\mathrm{PGE}_{2}$ via activation of the $\mathrm{EP} 3$ receptor (B.H. Koller, unpublished results). The relevant EP3expressing target cells have not been identified.

While prostanoids appear to promote acute inflammation in the majority of models, important exceptions have been observed. $\mathrm{PGE}_{2}$ has been reported to attenuate some acute inflammatory responses, in particular those initiated by mast cell degranulation. Raud and colleagues have shown that COX inhibition with indomethacin markedly potentiates antigen-induced plasma protein extravasation and leukocyte accumulation in sensitized hamsters (34). Moreover, $\mathrm{PGE}_{2} \mathrm{com}-$ pletely reversed the effects of indomethacin and reduced histamine release and plasma leakage in the absence of indomethacin. These results suggest that $\mathrm{PGE}_{2}$ can inhibit certain mast cell functions and are consistent with other reports showing enhanced histamine release from mast cells by indomethacin or suppression by E-type PGs (35).

There is also evidence to support a role for prostanoids in the resolution of inflammatory responses. Gilroy and colleagues showed in the rat carrageenan-induced pleurisy model that treatment with NSAIDs reduced the number of inflammatory cells and the formation of exudates present at 2 hours. How- 
ever, by 48 hours, when inflammation had largely resolved in the controls, the number of inflammatory cells had increased in the indomethacin-treated group (1). Similar findings have been observed in the air pouch model, where resolution of inflammation occurs more slowly in COX-2-deficient animals than in COX-1-deficient or wild-type controls (30).

Prostanoids also play a key role in pain associated with acute inflammation, and inhibition of these pathways has been an important therapeutic application for NSAIDs. Experiments with prostanoid receptor-deficient mice have provided new insights into the signaling pathways that mediate inflammatory pain, but these studies have also uncovered unexpected complexity in the mechanisms involved. Studies by Murata and colleagues showed that inflammatory pain responses are significantly reduced in mice lacking prostacyclin (IP) receptors, suggesting that IP receptors play a major role in the prostanoid-dependent component of pain (32). While these findings were somewhat unexpected, they are consistent with the previous demonstration of abundant expression of the IP receptor in neurons of the dorsal root ganglion (36). More recently, Stock and associates reported reduced responses to inflammatory pain in EP1-deficient mice (37). This inhibition in pain was virtually identical to that achieved through pharmacological interruption of PG synthesis in wild-type mice using an NSAID and was very similar to the magnitude of pain reduction seen in the IP-deficient mice. While the reasons for these apparently conflicting results is not clear, the differences in genetic background between the IP- and EP1-deficient mouse lines is one factor that may contribute. Alternatively, it is possible that signals from both the EP1 and IP receptors contribute to inflammatory pain and that the absence of either receptor is sufficient to attenuate the response. The observation that IP and EP1 receptors are coexpressed in dorsal root ganglia is consistent with this possibility (36). These data implicate EP1 and IP receptors as potential therapeutic targets for inflammatory pain.

Fever is another component of acute inflammation in which prostanoids may play a causal role. It is well established that COX inhibition reduces the fever associated with acute inflammation. Recent studies have shown that the febrile response to LPS is ameliorated in COX-2- but not COX-1-deficient mice, findings consistent with studies suggesting that COX-2 plays a dominant role in fever production (38). A number of lines of evidence suggest that the prostanoid responsible for fever production is $\mathrm{PGE}_{2}$, and studies using EPdeficient mice have shown that the febrile response to $\mathrm{PGE}_{2}, \mathrm{IL}-1 \beta$, and LPS occurs through the action of $\mathrm{PGE}_{2}$ on the EP3 receptor (39).

Chronic inflammation. High levels of prostanoids are observed in chronic inflammatory lesions, and several experimental approaches have been used to identify a role for these mediators in allergic rhinitis, asthma, rheumatoid arthritis, and inflammatory bowl disease. The mechanisms by which prostanoids can modulate these responses and contribute to disease do not differ in principle from those of an acute response: they can alter the response of both the host tissue and the recruited inflammatory cells. Prostanoids have been proposed to act on immune effector cells at any of several levels, and there is evidence that they can modulate the development, function, and survival of these cells. Again, the cells of the inflamed tissue or organ can also produce and respond to the prostanoids, providing an additional mechanism by which these lipid mediators may affect the course of the chronic inflammatory response. For example, prostanoids can alter the production of cytokines by epithelial cells and alter the expression of class II MHC antigens by antigen-presenting cells, thus modulating the course and resolution of the response (40).

Animal models of disease. Mice deficient in COX-isoenzymes and prostanoid receptors have been evaluated in several animal models of disease (Table 1). In a collagen-induced arthritis model, mice deficient in COX-2 display significant reductions in synovial inflammation and joint destruction, whereas arthritis in COX1-deficient mice is indistinguishable from controls (41). These data strongly suggest that prostanoids generated by COX-2 promote inflammation in this model and are consistent with the beneficial effects of COX-2 inhibitors in arthritis.

In contrast, in models of inflammatory colitis and allergic airway disease, an overall anti-inflammatory role for prostanoids has been suggested. Morteau and colleagues found that colitis induced by low-dose dextran sodium sulfate (DSS) is more severe in COX-2and, to a lesser extent, COX-1-deficient animals than in wild-type mice (42). Moreover, high-dose DSS is fatal in $50 \%$ of mice lacking either COX isoenzyme, whereas none of the control mice died following treatment. These findings are consistent with the reported exacerbation of inflammatory bowel disease in patients receiving NSAIDs.

Gavett and colleagues studied the inflammatory response of COX-1- and COX-2-deficient mice in the ovalbumin-induced asthma model (43). Lung inflammation, airway reactivity, and IgE levels were significantly enhanced in COX-1- and, to a lesser extent, COX2-deficient mice compared with wild-type controls. As expected, $\mathrm{PGE}_{2}$ levels were significantly reduced in COX-deficient mice and were substantially lower in COX-1- than in COX-2-deficient animals. Peebles and colleagues obtained similar results by treating mice with indomethacin; mice treated with this drug developed increased lung inflammation and tended to have higher serum IgE levels (44). Moreover, levels of IL-5 and 
IL-13 were significantly higher in mice treated with indomethacin. These data suggest that prostanoids can inhibit the development of the Th2 response in vivo, which is considered central to the pathogenesis of allergic inflammation. However, since leukotriene levels are higher in COX-deficient and indomethacin-treated mice than in controls, it remains undetermined whether increased inflammation might be secondary to shunting of AA toward the lipoxygenase pathway rather than direct inhibition of prostanoid synthesis.

Defining the contribution of individual prostanoids in complex inflammatory diseases has been aided by the development of mice lacking individual prostanoid receptors. In one such study, Matsuoka and colleagues examined the contribution of the $\mathrm{G}_{\mathrm{s}}{ }^{-}$ coupled DP receptor in the ovalbumin-induced asthma model (45). In contrast to COX-deficient animals, mice lacking the DP receptor show reduced disease. This finding is consistent with the well-established proinflammatory role of $\mathrm{PGD}_{2}$ in asthma, but the mechanism by which $\mathrm{PGD}_{2}$ mediates its proinflammatory actions is not clear. Since DP receptor activation in most cells examined to date leads to increased intracellular cAMP levels, $\mathrm{PGD}_{2}$ would be expected to attenuate the function of immune cells. Identification of a second receptor for $\mathrm{PGD}_{2}(\mathrm{CRTH} 2)$ that can couple to $G_{i}$ may, in part, explain the proinflammatory actions of this lipid mediator (8). Taken together, the results from COX-deficient and DP-deficient mice support a model in which both pro- and anti-inflammatory prostanoids are produced during chronic inflammation. This single example perhaps best illustrates the challenge awaiting us as we continue to dissect the role of individual lipid mediators in vivo.

\section{Conclusions}

Prostanoids play an important modulatory role in the immune response through complex interactions with leukocytes and parenchymal cells in the inflamed organ. They can produce both pro- and anti-inflammatory actions depending upon the inflammatory stimulus, the predominant prostanoid produced, and the profile of prostanoid receptor expression. Mice deficient in individual receptors and combinations of receptors, together with more specific pharmacological reagents, will allow the dissection of the role of these receptors in various models of inflammatory diseases. These studies should also determine whether other effector pathways such as modulation of PPAR $\gamma$ activity or direct actions of the cyclopentane prostanoids on the NF- $\kappa \mathrm{B}$ activity play any role in shaping immune responses in vivo. Uncovering the precise actions of individual prostanoids and their signal transduction pathways should yield important information about the biological significance of these lipid mediators in inflammation and may uncover new, more specific therapeutic targets for a number of diseases.

\section{Acknowledgments}

We apologize to those authors whose work could not be cited due to length limitations. We are indebted to J.L. Goulet, M.-T. Nguyen, L.P. Audoly, A.M. Latour, J.N. Snouwaert, and a series of graduate and undergraduate students and technicians who over the past years have generated, maintained, and studied the prostanoid-deficient mouse lines, and to R.J. Griffiths and his colleagues at Pfizer for encouraging and supporting our endeavors in this complex area of biology. Supported by grants from the NIH (HL04280 to S.L. Tilley, PO1-DK38108 to B.H. Koller and T.M. Coffman, and HL58554 to B.H. Koller).

1. Gilroy, D.W., et al. 1999. Inducible cyclooxygenase may have anti-inflammatory properties. Nat. Med. 5:698-701.

2. Smith, W.L., and DeWitt, D.L. 1996. Prostaglandin endoperoxide H synthases-1 and -2. Adv. Immunol. 62:167-215.

3. McAdams, B.F., et al. 2000. Effect of regulated expression of human cyclooxygenase isoforms on eicosanoid and isoeicosanoid production in inflammation. J. Clin. Invest. 105:1473-1482.

4. Reddy, S.T., and Herschman, H.R. 1997. Prostaglandin synthase-1 and prostaglandin synthase- 2 are coupled to distinct phospholipases for the generation of prostaglandin $\mathrm{D}_{2}$ in activated mast cells. J. Biol. Chem. 272:3231-3237.

5. Naraba, H., et al. 1998. Segregated coupling of phospholipases $A_{2}$, cyclooxygenases, and terminal prostanoid synthases in different phases of prostanoid biosynthesis in rat peritoneal macrophages. J. Immunol. 160:2974-2982.

6. Jakobsson, P.-J., Thoren, S., Morgenstern, R., and Samuelsson, B. 1999. Identification of human prostaglandin E synthase: a microsomal, glutathione-dependent, inducible enzyme, constituting a potential novel drug target. Proc. Natl. Acad. Sci. USA. 96:7220-7225.

7. Penglis, P.S., Cleland, L.G., Demasi, M., Caughey, G.E., and James, M.G. 2000. Differential regulation of prostaglandin $\mathrm{E}_{2}$ and thromboxane $\mathrm{A}_{2}$ production in human monocytes: implications for the use of cyclooxygenase inhibitors. J. Immunol. 165:1605-1611.

8. Hirai, H., et al. 2001. Prostaglandin $\mathrm{D}_{2}$ selectively induces chemotaxis in T helper type 2 cells, eosinophils, and basophils via seven-transmembrane receptor CRTH2. J. Exp. Med. 193:255-261.

9. Sugimoto, Y., Narumiya, S., and Ichikawa, A. 2000. Distribution and function of prostanoid receptors: studies from knockout mice. Prog. Lipid Res. 39:289-314.

10. Nishigaki, N., Negishi, M., and Ichikawa, A. 1996. Two Gs-coupled prostaglandin E receptor subtypes, EP2 and EP4, differ in desensitization and sensitivity to the metabolic inactivation of the agonist. Mol. Pharmacol. 50:1031-1037.

11. Kennedy, C., et al. 1999. Salt-sensitive hypertension and reduced fertility in mice lacking the prostaglandin EP2 receptor. Nat. Med. 5:217-220.

12. Tilley, S.L., et al. 1999. Reproductive failure and reduced blood pressure in mice lacking the EP2 prostaglandin $\mathrm{E}_{2}$ receptor. J. Clin. Invest. 103:1539-1545.

13. Nguyen, M., et al. 1997. The prostaglandin receptor $\mathrm{EP}_{4}$ triggers remodeling of the cardiovascular system at birth. Nature. 390:78-81.

14. Ushikubi, F., et al. 1993. Thromboxane $\mathrm{A}_{2}$ receptor is highly expressed in mouse immature thymocytes and mediates DNA fragmentation and apoptosis. J. Exp. Med. 178:1825-1830.

15. Remuzzi, G., et al. 1994. Thromboxane $A_{2}$ receptor blocking abrogates donor-specific unresponsiveness to renal allografts induced by thymic recognition of major histocompatibility allopeptides. J. Exp. Med. 180:1967-1972.

16. Goetzl, E.J., An, S., and Zeng, L. 1995. Specific suppression by prostaglandin $\mathrm{E}_{2}$ of activation-induced apoptosis of human CD4+CD8+ T lymphoblasts. J. Immunol. 154:1041-1047.

17. Rocca, B., et al. 1999. Distinct roles of prostaglandin $H$ synthases 1 and 2 in T-cell development. J. Clin. Invest. 103:1469-1477.

18. Chouaib, S., Welte, K., Mertelsmann, R., and Dupont, B. 1985. Prostaglandin $\mathrm{E}_{2}$ acts at two distinct pathways of T lymphocyte activation: inhibition of interleukin 2 production and down-regulation of transferrin receptor expression. J. Immunol. 135:1172-1179.

19. Roper, R.L., Ludlow, J.W., and Phipps, R.P. 1994. Prostaglandin $E_{2}$ 
inhibits B lymphocyte activation by a cAMP-dependent mechanism: PGE-inducible regulatory proteins. Cell. Immunol. 154:296-308.

20. Pene, J., et al. 1988. IgE production by normal human lymphocytes is induced by interleukin 4 and suppressed by interferons $\gamma$ and $\alpha$ and prostaglandin $\mathrm{E}_{2}$. Proc. Natl. Acad. Sci. USA. 85:6880-6884.

21. Ruiz, P., Spurney, R., Coffman, T., and Viciana, A. 1992. Thromboxane augmentation of alloreactive $T$ cell function. Transplantation. 54:498-505.

22. Kaur, K., Harris, S.G., Padilla, J., Graf, B.A., and Phipps, R.P. 1999. Prostaglandin $\mathrm{E}_{2}$ as a modulator of lymphocyte mediated inflammatory and humoral responses. Adv. Exp. Med. Biol. 469:409-411.

23. Khan, M.M. 1995. Regulation of IL-4 and IL-5 secretion by histamine and $\mathrm{PGE}_{2}$. Adv. Exp. Med. Biol. 383:35-42.

24. Kliewer, S.A., et al. 1995 . A prostaglandin $J_{2}$ metabolite binds peroxisome proliferator-activated receptor gamma and promotes adipocyte differentiation. Cell. 83:813-819.

25. Narumiya, S., and FitzGerald, G.A. 2001. Genetic and pharmacological analysis of prostanoid receptor function. J. Clin. Invest. 108:25-30.

26. Jiang, C., Ting, A., and Seed, B. 1998. PPAR- $\gamma$ agonists inhibit production of monocyte inflammatory cytokines. Nature. 391:82-86.

27. Ricote, M., Li, A.C., Willsons, T.M., Kelly, C.J., and Glass, C.K. 1998. The peroxisome proliferator-activated receptor- $\gamma$ is a negative regulator of macrophage activation. Nature. 391:79-82.

28. Rossi, A., et al. 2000. Anti-inflammatory cyclopentenone prostaglandins are direct inhibitors of IkappaB kinase. Nature. 403:103-108.

29. Straus, D.S., et al. 2000. 15-Deoxy- $\Delta^{12,1}$ - prostaglandin $J_{2}$ inhibits multiple steps in the NF-кB signaling pathway. Proc. Natl. Acad. Sci. USA. 97:4844-4849.

30. Langenbach, R., Loftin, C.D., Lee, C., and Tiano, H. 1999. Cyclooxygenase-deficient mice. A summary of their characteristics and susceptibilities to inflammation and carcinogenesis. Ann. NY. Acad. Sci. 889:52-61.

31. Muller-Decker, K., et al. 1999. Prostaglandin-H-synthase isozyme expression in normal and neoplastic human skin. Int. J. Cancer. 82:648-656.

32. Murata, T., et al. 1997. Altered pain perception and inflammatory response in mice lacking prostacyclin receptor. Nature. 388:678-682.

33. Audoly, L.P., et al. 1999. Identification of specific EP receptors responsible for the hemodynamic effects of PGE 2. Am. J. Physiol. 277:H924-H930.

34. Raud, J., Dahlen, S.-E., Sydbom, A., Lindbom, L., and Hedqvist, P. 1988. Enhancement of acute allergic inflammation by indomethacin is reversed by prostaglandin $\mathrm{E}_{2}$ : apparent correlation with in vivo modulation of mediator release. Proc. Natl. Acad. Sci. USA. 85:2315-2319.
35. Hitchcock, M. 1978. Effect of inhibitors of prostaglandin synthesis and prostaglandin $E_{2}$ and $F_{2 \alpha}$ on the immunologic release of mediators of inflammation from actively sensitized guinea-pig lung. J. Pharmacol. Exp. Ther. 207:630-640.

36. Oida, H., et al. 1995. In situ hybridization studies of prostacyclin receptor mRNA expression in various mouse organs. Br. J. Pharmacol. 116:2828-2837.

37. Stock, J.L., et al. 2001. The prostaglandin $\mathrm{E}_{2} \mathrm{EP1}$ receptor mediates pain perception and regulates blood pressure. J. Clin. Invest. 107:325-331.

38. Li, S., et al. 1999. The febrile response to lipopolysaccharide is blocked in cyclooxygenase- $2^{-/-}$, but not in cyclooxygenase $-1^{-/-}$mice. Brain Res. 825:86-94.

39. Ushikubi, F., et al. 1998. Impaired febrile response in mice lacking the prostaglandin $\mathrm{E}$ receptor subtype $\mathrm{EP}_{3}$. Nature. 395:281-284.

40. Arvind, P., et al. 1995. $\mathrm{PGE}_{2}$ down-regulates the expression of HLA-DR antigen in human colon adenocarcinoma cell lines. Biochemistry. 34:5604-5609.

41. Meyers, L.K., et al. 2000. The genetic ablation of cyclooxygenase 2 prevents the development of autoimmune arthritis. Arthritis Rheum. 43:2687-2693.

42. Morteau, O., et al. 2000. Impaired mucosal defense to acute colonic injury in mice lacking cyclooxygenase-1 or cyclooxygenase-2. J. Clin. Invest. 105:469-478.

43. Gavett, S.H., et al. 1999. Allergic lung responses are increased in prostaglandin H synthase-deficient mice. J. Clin. Invest. 104:721-732.

44. Peebles, R.S., et al. 2000. Cyclooxygenase inhibition increases interleukin 5 and interleukin 13 production and airway hyperresponsiveness in allergic mice. Am. J. Respir. Crit. Care Med. 162:676-681.

45. Matsuoka, T., et al. 2000. Prostaglandin $\mathrm{D}_{2}$ as a mediator of allergic asthma. Science. 287:2013-2017.

46. Langenbach, R., et al. 1995. Prostaglandin synthase 1 gene disruption in mice reduces arachidonic acid-induced inflammation and indomethacin-induced gastric ulceration. Cell. 83:483-492.

47. Morham, S.G., et al. 1995. Prostaglandin synthase 2 gene disruption causes severe renal pathology in the mouse. Cell. 83:473-482.

48. Dinchuk, J.E., et al. 1995. Renal abnormalities and an altered inflammatory response in mice lacking cyclooxygenase II. Nature. 378:406-409.

49. Wallace, J.L., et al. 1998. Cyclooxygenase 1 contributes to inflammatory responses in rats and mice: implications for gastrointestinal toxicity. Gastroenterology. 115:101-109. 